\title{
Clinical significance and prognostic value of SOX7 expression in liver and pancreatic carcinoma
}

\author{
JIAN WANG, SHENGMIN ZHANG, JIAMIAN WU, ZHUOCAI LU, JIANRONG YANG, \\ HONGSHENG WU, HAO CHEN, BO LIN and TIANSHENG CAO \\ Department of General Surgery, Huadu District People's Hospital, \\ Southern Medical University, Guangzhou, Guangdong 510800, P.R. China
}

Received January 26, 2016; Accepted January 17, 2017

DOI: $10.3892 / \mathrm{mmr} .2017 .6660$

\begin{abstract}
Sex determining region Y-box 7 (SOX7) is known to function as a tumor suppressor in a number of types of cancer; however, its role in liver and pancreatic carcinoma remains unclear. The present study investigated the association between SOX7 expression and the clinical pathology of these carcinomas, in particular if SOX7 expression may be used to predict recurrence and patient prognosis following radical resection of liver and pancreatic carcinoma. SOX7 expression in human liver and pancreatic carcinoma was detected by immunohistochemical analyses and validated using mRNA data from a high-throughput sequencing dataset from The Cancer Genome Atlas (TCGA). SOX7 expression was significantly downregulated in liver and pancreatic carcinoma relative to the adjacent benign tissues [immunoreactivity scores: Liver carcinoma $(3.53 \pm 1.57)$ vs. benign $(7.00 \pm 0.00)$, $\mathrm{P}<0.001$; and pancreatic carcinoma $(2.39 \pm 1.88)$ vs. benign (4.80 \pm 0.45$), \mathrm{P}=0.005]$. In addition, downregulation of SOX7 was significantly associated with advanced stage liver carcinoma, and the primary pathological tumor stage and regional lymph node stages. These findings were further validated in the TCGA dataset. However, SOX7 down regulation was closely associated with the only pathological grade in pancreatic patients. Kaplan-Meier analyses revealed significant differences in overall and disease-free survival between patients with high and low levels of SOX7 expression. In addition, a multivariate analysis with Cox regression indicated that SOX7 may be an independent predictor of disease-free survival. The results indicate that $\mathrm{SOX} 7$ may inhibit the progression of liver carcinoma and that SOX7 downregulation may accurately predict poor prognosis in liver carcinoma patients.
\end{abstract}

Correspondence to: Mr. Tiansheng Cao, Department of General Surgery, Huadu District People's Hospital, Southern Medical University, 48 Xinhua Road, Xinhua Street, Huadu, Guangzhou, Guangdong 510800, P.R. China

E-mail: caotiansheng2088@sina.com

Key words: sex determining region Y-box 7, liver carcinoma, pancreatic carcinoma, clinicopathological feature, prognosis

\section{Introduction}

Malignant digestive tumors are a growing concern in human health. In China, the incidence and mortality rates associated with malignant digestive tumors appear to be steadily increasing. Among the different types of digestive malignant tumors, liver and pancreatic cancers remain the most difficult to diagnosis and treat. New treatments are continuously developed, however, the overall survival rate has only modestly improved over the past decade. Liver cancer is the fifth most common cancer in the world and the third leading cause of cancer-associated mortality worldwide $(1,2)$. Pancreatic cancer is the fourth leading cause of cancer-associated mortality globally (3). The World Health Organization classifies primary liver cancers into hepatocellular carcinoma (HCC), intrahepatic cholangiocarcinoma (ICC) and combined hepatocellular-cholangiocarcinoma (4). HCC represents the major histological subtype of primary liver cancer, however the incidence of ICC, the second most frequent type of liver cancer, has increased dramatically over the past two decades (5).

Sex determining region Y-box 7 (SOX7), SOX17 and SOX18, belong to the SOX group F subfamily (6). SOX7 encodes a high mobility group box transcription factor and has been implicated in a number of developmental processes (7), including hematopoiesis (8), cardiogenesis (9), vasculogenesis (10), endoderm differentiation (11) and myogenesis (12). As a transcription factor, SOX7 activates target genes and regulates the Wnt/ $\beta$-catenin signaling pathway (13). SOX7 may compete with T-cell factor (TCF)/lymphoid enhancing factor (LEF) activity by interacting with $\beta$-catenin and inhibiting the expression of target genes. Previous studies have observed that SOX7 expression is deregulated in different human cancer types compared with SOX7 in normal tissues (14-16). Katoh (17) revealed that SOX7 expression levels were increased in a number of pancreatic, gastric and esophageal cancer cell lines, and also in 4 cases of primary gastric cancer. The majority of other studies investigating SOX7 expression in cancer have demonstrated a downregulation of SOX7 expression levels in a variety of human cancer types, including breast (18), lung (15), colon (14) and prostate (19). A decrease in SOX7 expression levels has been associated with poor prognosis in lung adenocarcinoma (15) and prostate cancer (19). However, the clinical significance of SOX7 expression in liver 
and pancreatic cancers remains unclear. Thus, the aim of the present study was to investigate the association between SOX7 expression and tumor progression and prognosis in patients with liver and pancreatic cancer.

\section{Materials and methods}

Patients and tissue samples. The present study was approved by the Ethics Committee of Guangzhou First People's Hospital, Guangzhou Medical University (Guangdong, China). All procedures performed involving human participants were in accordance with the ethical standards of the institutional and/or national research committee and with the 1964 Helsinki declaration and its later amendments or comparable ethical standards. All applicable international, national, and/or institutional guidelines for the care and use of animals were followed. Informed consent was obtained from all individual participants included in the study. Tissue microarrays (TMAs) were obtained from Xi'an Ailina Biotechnology Co., Ltd. (Xi'an, China). The HCC TMA (cat. no. LV802a) used in the present study contained 78 primary $\mathrm{HCC}$ and 2 adjacent non-cancerous liver tissues, with the age of the donors ranging between 18 and 73 years (mean age, 51.78 years). The ICC TMA (cat. no. LV1004) used contained 100 primary ICC tissues that were collected from 42 female and 58 male patients (age range, 27 to 74 years; mean age, 54.72 years), and 5 adjacent normal tissues were recruited from the Huadu District People's Hospital (Guangzhou, China) in September 2015 as the ICC TMA did not contain any normal tissues. Of the HCC and ICC TMA samples, 1 sample from each TMA was removed, and 3 samples were interstitial samples, which were also not analyzed. Therefore, the total number of cancer samples analyzed in HCC and ICC TMAs was 173. The pancreatic microarray (cat. no. PA961b) contained 91 pancreatic cancer tissues and 5 normal pancreatic tissues that were collected from patients aged 21 to 78 years old (mean age, 51.87 years). As 3 pancreatic cancer samples could not be evaluated and scored for the percentage of staining, a total of 88 pancreatic cancer samples were analyzed for $\mathrm{SOX} 7$ protein expression.

To investigate the expression of SOX7 at the mRNA level, the Cancer Genome Atlas (TCGA) dataset, a large cancer dataset with high-throughput sequencing data for protein coding genes (mRNA), which includes 134 HCC tissues, was used (https://tcga-data.nci.nih.gov, liver hepatocellular carcinoma). Detailed information regarding the clinical features of the patients is presented in Table I.

Immunohistochemistry. The TMA slides were deparaffinized with xylene and then serially rehydrated with ethanol $(100,100$, 95, 95, 90, 80 and 70\%). Following brief proteolytic digestion using the IHC enzyme antigen retrieval agent (Wuhan Boster Biological Technology, Ltd., Wuhan, China) and peroxidase blocking with $3 \% \mathrm{H}_{2} \mathrm{O}_{2}$, the slides were incubated at $4^{\circ} \mathrm{C}$ overnight with a rabbit polyclonal primary antibody against $\mathrm{SOX} 7$ (dilution, 1:200; cat. no. sc-20,093; Santa Cruz Biotechnology, Inc., Dallas, TX, USA). Following washing with PBS to remove any unbound primary antibodies, the slides were incubated for $30 \mathrm{~min}$ with peroxidase-conjugated secondary antibody (HRP-labeled Goat Anti-Rabbit IgG; dilution, 1:1,000; cat. no. A0208; Beyotime Institute of Biotechnology Co., Ltd., Haimen, China) at room temperature. The specifically bound secondary antibody was detected using a DAKO EnVision detection system (Dako Diagnostics; Agilent Technologies, Inc., Santa Clara, CA, USA).

Evaluation of staining. Following counterstaining with hematoxylin, stained TMAs were scanned by Xi'an Ailina Biotechnology Co., Ltd. using a digital high resolution microscope scanner (Aperio ScanScope; Aperio Technologies, Inc., Vista, CA, USA) and viewed using ImageScope v.11 software (Aperio Technologies, Inc.). Protein expression was scored by two independent, experienced pathologists who were unaware of the clinical data and patient outcomes. The two sets of scores produced were compared and the discrepant scores were re-examined by the two pathologists to achieve a consensus score. The number of positively stained cells was counted in 10 representative microscopic fields, and the percentage of positively stained cells was calculated. Given the homogeneity of the target protein staining, the tumor specimens were scored in a semi-quantitative manner. The percentage scoring of immunoreactive tumor cells was as follows: 0, no staining; 1, weak; 2, moderate; and 3, strong. The specimens were also evaluated and scored for the percentage of staining as follows: $1,0-25 \% ; 2,25-50 \%$; $3,50-75 \%$; and 4, 75-100\%. A final immunoreactivity score (IRS) was obtained for each case by summing the percentage and the intensity scores. Protein expression levels were further analyzed by classifying the IRS values as low or high, based on the median IRS value.

Statistical analysis. SPSS 21.0 software (IBM SPSS, Armonk, NY, USA) was used for statistical analysis. Pearson's Chi-squared tests and Fisher's exact test were used to analyze the association between SOX7 protein expression and clinicopathological characteristics. Student's t-test was used to analyze the association between SOX7 mRNA expression and clinicopathological characteristics. Overall survival was analyzed using the Kaplan-Meier method, and the differences were assessed using a log-rank test. Univariate analysis comparisons [comparisons between gender, age, the clinical stage, the pathological tumor node metastasis (pTNM) stage and SOX7 mRNA expression] and multivariate survival comparisons were performed using Cox proportional hazard regression models. The relative risks of mortality were expressed as adjusted hazard ratios (HRs) with corresponding 95\% confidence intervals (CIs). $\mathrm{P}<0.05$ was considered to indicate a statistically significant difference.

\section{Results}

SOX7 protein expression is increased in human liver and pancreatic cancers. The present study investigated whether SOX7 expression is altered with clinical cancer specimens by analyzing liver and pancreatic TMAs (Table I). In these TMAs, the expression profile and localization of SOX7 in the 173 liver cancer tissues and 7 adjacent non-cancerous liver tissues (Fig. 1), and 88 pancreatic cancer tissues and 5 adjacent non-cancerous pancreatic tissues (Fig. 2) were examined by immunohistochemical analyses. 
Table I. Clinical features of the patients recruited in the present study.

\begin{tabular}{|c|c|c|c|}
\hline \multirow[b]{2}{*}{ Clinical feature } & \multicolumn{2}{|c|}{ Immunohistochemistry results } & \multirow[b]{2}{*}{ Liver TCGA dataset } \\
\hline & Liver & Pancreatic & \\
\hline Total no. of cancer cases & 178 & 91 & 134 \\
\hline \multicolumn{4}{|l|}{ Gender (n) } \\
\hline Male & 120 & 56 & 80 \\
\hline Female & 58 & 35 & 54 \\
\hline \multicolumn{4}{|l|}{ Age (n) } \\
\hline$<60$ & 124 & 71 & 67 \\
\hline$\geq 60$ & 54 & 20 & 67 \\
\hline Mean age (years) & $53.41 \pm 11.11$ & $52.70 \pm 9.96$ & $62.10 \pm 13.34$ \\
\hline \multicolumn{4}{|l|}{ Pathological grade (n) } \\
\hline$\leq 2$ & 77 & 51 & - \\
\hline$>2$ & 89 & 15 & - \\
\hline \multicolumn{4}{|l|}{ Clinical stage (n) } \\
\hline $\mathrm{I} / \mathrm{II}$ & 80 & 84 & - \\
\hline III/IV & 98 & 6 & - \\
\hline I & - & - & 50 \\
\hline II-IV & - & - & 75 \\
\hline \multicolumn{4}{|l|}{ pT stage (n) } \\
\hline $\mathrm{T} 1-\mathrm{T} 2$ & 91 & 34 & 53 \\
\hline $\mathrm{T} 3-\mathrm{T} 4$ & 86 & 56 & 81 \\
\hline \multicolumn{4}{|l|}{ pN stage (n) } \\
\hline No & 144 & 78 & 83 \\
\hline $\mathrm{N} 1 / \mathrm{N} 2$ & 33 & 12 & 3 \\
\hline Metastasis (n) & 3 & 2 & 3 \\
\hline Benign (n) & 7 & 5 & 0 \\
\hline
\end{tabular}

'-' indicates a lack of the relative information for these patients. Of the 134 patients in the TCGA dataset, 9 had no information regarding clinical stage. TCGA, The Cancer Genome Atlas; pT stage, primary tumor stage; pN stage, regional lymph node stage.

The SOX7 expression level in liver cancer tissues (Fig. 1A and B) was significantly lower than that observed in non-cancerous liver tissues [IRS: Liver cancer $(3.53 \pm 1.57)$ vs. benign (7.00 \pm 0.00$), \mathrm{P}<0.001$; Fig. $1 \mathrm{C}$ ). Immunohistochemistry revealed weak or moderate SOX7 staining in the cytoplasm of liver cancer cells (Fig. 1D-F) however, strong SOX7 staining was observed in the adjacent non-cancerous liver tissues (Fig. 1G). As observed in the cancerous liver tissues, SOX7 protein was expressed in the cytoplasm of pancreatic cancer cells and adjacent non-cancerous pancreatic tissue cells (Fig. 2A). However, the level of SOX7 expression was different between the two cancer types (Fig. 2B-E). In pancreatic cancer tissues the level of SOX7 expression was significantly lower than that observed in non-cancerous pancreatic tissues [IRS: Pancreatic cancer (2.39 \pm 1.88$)$ vs. benign $(4.80 \pm 0.45), \mathrm{P}=0.005$; Fig. $2 \mathrm{~B})$.

The immunostaining results were analyzed further using the limited quantity of clinical information regarding the liver and pancreatic TMAs of the donors (Tables II and III). The liver TMA results indicated that a low level of SOX7 expression is significantly associated with higher clinical stage $(\mathrm{P}<0.001)$, high primary pathological tumor $(\mathrm{pT})$ stage $(\mathrm{P}=0.006)$ and $\mathrm{a}$ high regional lymph node $(\mathrm{pN})$ stage $(\mathrm{P}=0.002)$ however, it was not associated with age $(\mathrm{P}=0.313)$, gender $(\mathrm{P}=0.328)$ or pathological grade $(\mathrm{P}=0.448)$. The pancreatic TMA results (Table III) indicated that a low level of SOX7 expression is significantly associated with a high pathological grade $(\mathrm{P}=0.026)$, however, it is not associated with age $(\mathrm{P}=0.378)$, gender $(\mathrm{P}=0.132)$, clinical stage $(\mathrm{P}=0.305)$, $\mathrm{pT}$ stage $(\mathrm{P}=0.718)$, $\mathrm{pN}$ stage $(\mathrm{P}=0.097)$ or pathological metastasis $(\mathrm{pM})$ stage $(\mathrm{P}=0.411)$.

Decreased SOX7 expression is associated with the aggressive progression and poor prognosis of human liver cancer in the TCGA dataset. To validate the results of the TMA cohort, a publicly available dataset was used. The TCGA dataset (https://tcga-data.nci.nih.gov), consists of high-throughput sequencing data for protein coding gene expression (mRNA) data from 134 liver cancer tissues. The data presented in Table II revealed that SOX7 downregulation is frequently identified in liver cancer tissues with an advanced clinical stage $(\mathrm{P}=0.045)$, pT stage $(\mathrm{P}=0.010), \mathrm{pN}$ stage $(\mathrm{P}=0.004)$ or $\mathrm{pM}$ stage $(\mathrm{P}=0.037)$. However, $\mathrm{SOX} 7$ expression was not significantly different when comparing patient gender (female vs. male, $\mathrm{P}=0.340$ ) or age ( $<60$ vs. $\geq 60$ years, $\mathrm{P}=0.978)$. 

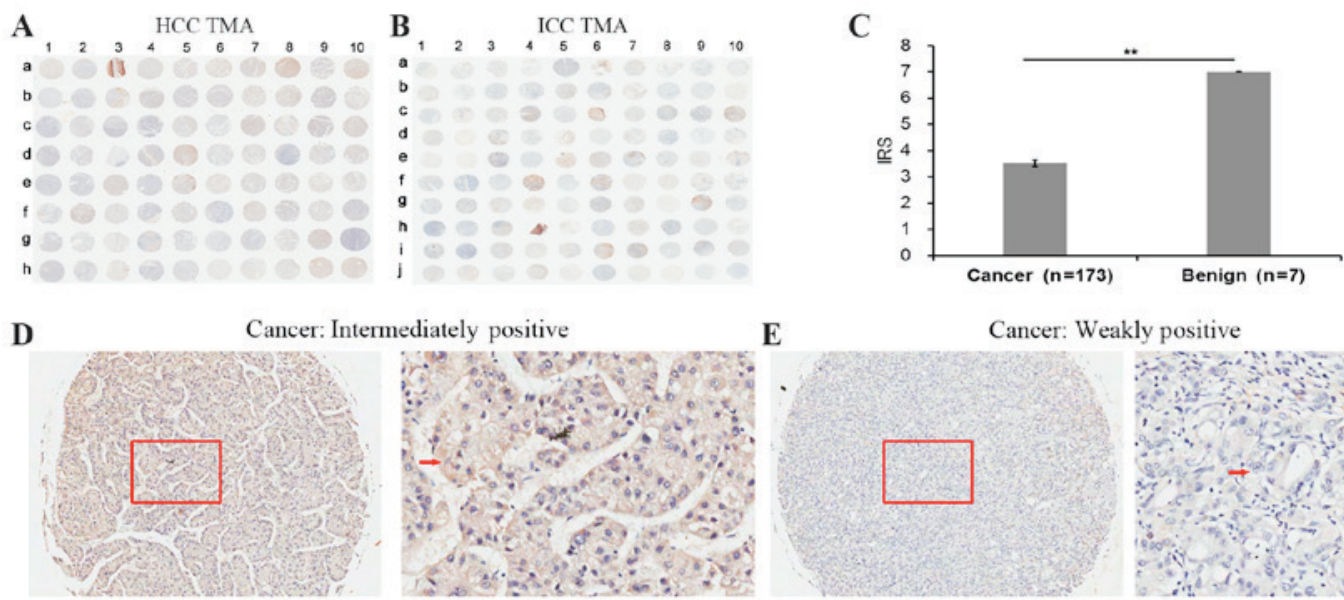

E Cancer: Weakly positive

$\mathbf{F}$

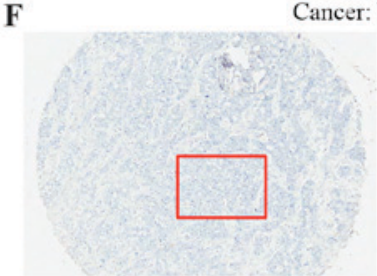

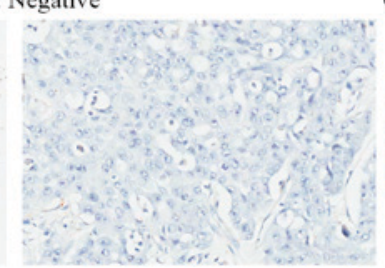
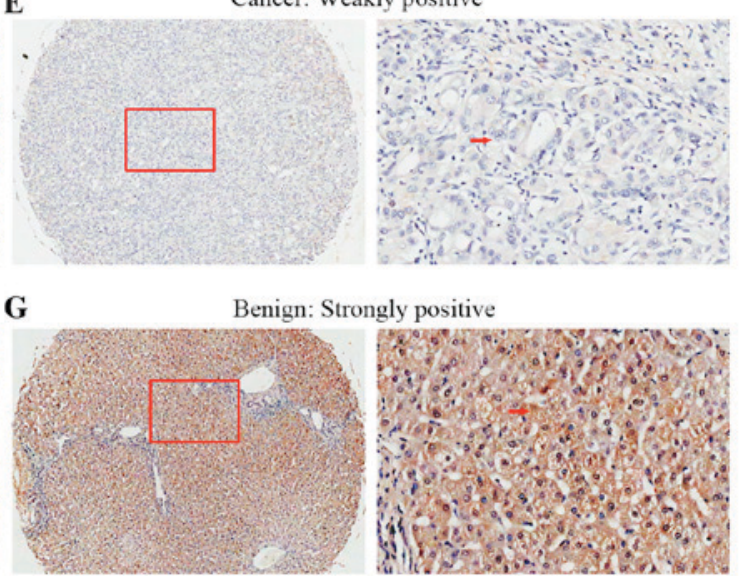

Figure 1. Immunohistochemical staining for SOX7 in liver cancer and adjacent non-cancerous liver tissues in a TMA. Immunohistochemistry staining for SOX7 in the (A) HCC and (B) ICC TMA cohorts. (C) IRS of SOX7 in liver cancer were lower than those observed in the adjacent normal liver tissues. IRS: ${ }^{* *} \mathrm{P}<0.001$, liver cancer $(3.53 \pm 1.57)$ vs. benign $(7.00 \pm 0.00)$. Data are presented as the mean \pm standard deviation. Immunohistochemistry staining indicated that SOX7 immunostaining primarily occurred in the cytoplasm and cellular membrane of liver cancer tissues cells. The intensities of SOX7 immunostaining were (D) intermediate, (E) weak and (F) negative, no strong staining was observed in cancer samples. Left image, magnification x100; right image, magnification $\mathrm{x} 400$; the red squares indicate the area shown at higher magnification. (G) Strongly positive immunohistochemistry staining in the adjacent non-cancerous liver tissue cells. Red arrows indicate positively-stained cells. SOX7, sex determining region Y-box 7; HCC, hepatocellular carcinoma; TMA, tissue microarrays; ICC, intrahepatic cholangiocarcinoma; IRS, immunoreactivity scores.

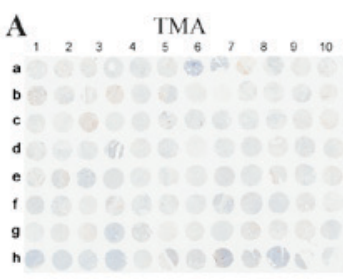

B

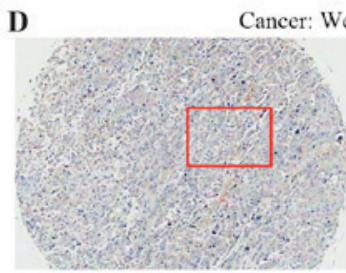

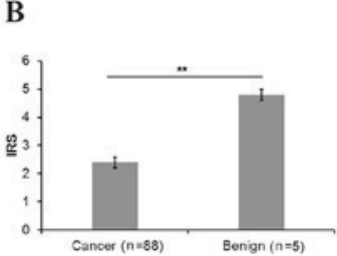

akly positive

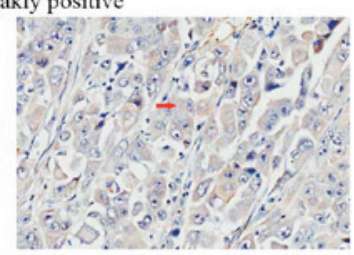

C

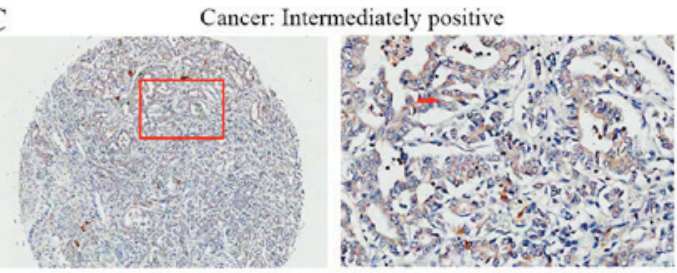

E

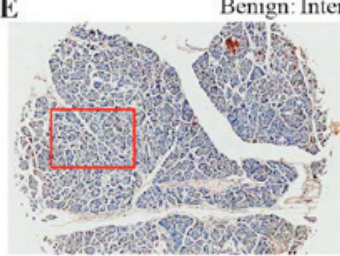

ermediately positive

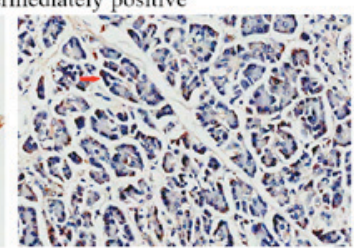

Figure 2. Immunohistochemical staining for SOX7 in pancreatic cancer and adjacent non-cancerous pancreatic tissues in a TMA. (A) Immunohistochemistry staining for SOX7 in the pancreatic cancer TMA cohort. (B) IRS of SOX7 in pancreatic cancer were lower than those observed in the adjacent normal pancreatic tissues IRS: ${ }^{* *} \mathrm{P}=0.005$, pancreatic cancer $(2.39 \pm 1.88)$ vs. benign $(4.80 \pm 0.45)$. Data were presented as the mean \pm standard deviation. ${ }^{* *} \mathrm{P}<0.01$. The immunohistochemistry staining indicated that SOX7 immunostaining primarily occurred in the cytoplasm and cellular membrane of pancreatic cancer tissue cells. The intensities of SOX7 immunostaining observed were (C) intermediate and (D) weak, with no strong staining observed in cancer tissues. Left image, magnification x100 and right image, magnification x400; the red squares indicate the area shown at higher magnification. (E) Intermediately immunohistochemistry staining in adjacent non-cancerous pancreatic tissue cells. SOX7, sex determining region Y-box 7; TMA, tissue microarrays; IRS, immunoreactivity scores.

To demonstrate the prognostic value of detecting SOX7 expression levels in patients with liver cancer, the Kaplan-Meier method was performed to compare the prognosis among patients with high and low SOX7 expression levels (Fig. 3). The mean level of SOX7 mRNA expression in the TCGA dataset was used as the cut-off to divide patients with liver cancer into the high and low SOX7 expression groups. The patients with liver cancer and low SOX7 expression levels had a poorer prognosis than those with high SOX7 expression levels $(\mathrm{P}=0.032 ; \mathrm{Fig} .3 \mathrm{~A})$, and their length of biochemical recurrence (BCR)-free survival time was significantly shorter than that of patients with high $\mathrm{SOX} 7$ expression levels ( $\mathrm{P}=0.009$; Fig. $3 \mathrm{~B}$ ). 
Table II. Associations between SOX7 expression and clinicopathological characteristics in patients with liver cancer.

\begin{tabular}{|c|c|c|c|c|c|c|c|}
\hline \multirow[b]{2}{*}{ Clinical feature } & \multicolumn{4}{|c|}{ IRS of SOX7 protein expression in the cohort } & \multicolumn{3}{|c|}{$\begin{array}{l}\text { SOX7 mRNA expression } \\
\text { in TCGA database }\end{array}$} \\
\hline & $\begin{array}{l}\text { No. of } \\
\text { total cases }\end{array}$ & $\begin{array}{l}\text { No. of low } \\
\text { (\% of total no.) }\end{array}$ & $\begin{array}{l}\text { No. of high } \\
\text { (\% of total no.) }\end{array}$ & P-value & $\begin{array}{l}\text { No. of } \\
\text { total cases }\end{array}$ & $\overline{\mathrm{x}} \pm \mathrm{s}$ & P-value \\
\hline Tissue & & & & $0.022^{\mathrm{a}}$ & & & - \\
\hline Cancer & 173 & $118(68.2)$ & $55(31.8)$ & & 134 & $144.81 \pm 166.64$ & \\
\hline Benign & 7 & $3(42.9)$ & $4(57.1)$ & & - & - & \\
\hline Age & & & & 0.313 & & & 0.978 \\
\hline$<60$ & 120 & $79(65.8)$ & $41(34.2)$ & & 67 & $144.41 \pm 164.23$ & \\
\hline$\geq 60$ & 53 & $39(73.6)$ & $14(26.4)$ & & 67 & $145.21 \pm 170.26$ & \\
\hline Gender & & & & 0.328 & & & 0.340 \\
\hline Male & 117 & $77(65.8)$ & $40(34.2)$ & & 80 & $156.13 \pm 187.80$ & \\
\hline Female & 56 & $41(73.2)$ & $15(26.8)$ & & 54 & $128.03 \pm 128.98$ & \\
\hline Pathological grade & & & & 0.448 & & & - \\
\hline$\leq 2$ & 77 & $55(71.4)$ & $22(28.6)$ & & - & - & \\
\hline$>2$ & 85 & $56(65.9)$ & $29(34.1)$ & & - & - & \\
\hline Clinical stage & & & & $<0.001^{\mathrm{b}}$ & & & $0.012^{\mathrm{a}}$ \\
\hline $\mathrm{I} / \mathrm{II}$ & 75 & $27(36.0)$ & $48(64.0)$ & & - & - & \\
\hline III/IV & 98 & $91(92.9)$ & 7 (7.1) & & - & - & \\
\hline I & - & - & - & - & 50 & $187.66 \pm 227.94$ & \\
\hline II-IV & - & - & - & - & 75 & $111.95 \pm 96.99$ & \\
\hline pT stage & & & & $0.006^{\mathrm{b}}$ & & & $0.010^{\mathrm{a}}$ \\
\hline $\mathrm{T} 1$ & 54 & $29(53.7)$ & $25(46.3)$ & & 53 & $190.64 \pm 230.06$ & \\
\hline $\mathrm{T} 2-\mathrm{T} 4$ & 119 & $89(74.8)$ & $30(25.2)$ & & 81 & $114.82 \pm 97.27$ & \\
\hline pN stage & & & & $0.002^{\mathrm{b}}$ & & & $0.004^{\mathrm{b}}$ \\
\hline No & 139 & 87 (62.6) & $52(37.4)$ & & 83 & $156.05 \pm 178.34$ & \\
\hline N1 & 33 & $30(90.9)$ & $3(9.1)$ & & 3 & $50.42 \pm 32.27$ & \\
\hline pM stage & & & & 0.231 & & & $0.037^{\mathrm{a}}$ \\
\hline M0 & 169 & $114(67.5)$ & $55(32.5)$ & & 98 & $143.94 \pm 168.04$ & \\
\hline M1 & 3 & $3(100.0)$ & $0(0.0)$ & & 3 & $100.87 \pm 16.91$ & \\
\hline
\end{tabular}

${ }^{\mathrm{a}} \mathrm{P}<0.05$ and ${ }^{\mathrm{b}} \mathrm{P}<0.01$. '-' indicates that there is a lack of the relative information for these patients. SOX7, sex determining region Y-box 7 ; IRS, immunoreactivity scores; TCGA, The Cancer Genome Atlas; pT stage, primary tumor stage; pN stage, regional lymph node stage; pM, metastasis stage.

When the liver patients with metastases were removed from the analysis, pairwise comparisons revealed no significant differences in the lengths of BCR-free survival time between the two groups $(\mathrm{P}=0.105$; Fig. $3 \mathrm{C})$; however, $\mathrm{SOX} 7$ expression was able to stratify the non-metastatic patients into those with a high risk and a low risk of $\mathrm{BCR}(\mathrm{P}=0.006$; Fig. 3D).

SOX7 expression serves as an independent predictor of disease-free survival in patients with liver cancer. The present study also investigated whether SOX7 expression levels were an effective independent predictor of disease-free survival in the patients with liver cancer from the TCGA dataset by performing univariate and multivariate analyses. Patients with liver cancer and low tumor levels of SOX7 expression had significantly lower disease-free survival rates than those with high tumor levels of SOX7 expression (Table IV). The SOX7 expression level (HR: 0.314; 95\% CI: 0.130-0.760; $\mathrm{P}=0.010$ ) and the pM stage (HR: $10.922 ; 95 \% \mathrm{CI}: 1.897-62.883 ; \mathrm{P}=0.007$ ) were able to serve as independent predictors for disease-free survival (Table IV). This suggests that the SOX7 expression level may be an independent predictor for disease-free survival in patients with liver cancer.

\section{Discussion}

The majority of digestive malignant tumors, including liver and pancreatic cancers, are diagnosed at an advanced stage, when clinical deterioration has occurred and curative therapies are no longer effective $(20,21)$. Therefore, the early detection of HCC and pancreatic cancers, particularly at a time when curative surgery can still be performed, is potentially the best method to improve patient prognosis $(21,22)$. Thus, screening novel tumor biomarkers is important for the prevention, diagnosis and targeted therapy of liver and pancreatic cancers. The 
Table III. Association between SOX7 protein expression and clinicopathological characteristics in patients with pancreatic carcinoma.

\begin{tabular}{|c|c|c|c|}
\hline Clinical feature & No. of total cases & SOX7 protein expression $(\overline{\mathrm{x}} \pm \mathrm{s})$ & P-value \\
\hline Tissue & & & $0.002^{\mathrm{a}}$ \\
\hline Cancer & 88 & $1.89 \pm 1.87$ & \\
\hline Normal & 5 & $4.80 \pm 0.45$ & \\
\hline Age (years) & & & 0.378 \\
\hline$<50$ & 29 & $2.14 \pm 2.22$ & \\
\hline$\geq 50$ & 59 & $1.76 \pm 1.68$ & \\
\hline Gender & & & 0.132 \\
\hline Males & 54 & $2.26 \pm 2.39$ & \\
\hline Female & 34 & $1.65 \pm 1.42$ & \\
\hline Pathological grade & & & $0.026^{\mathrm{b}}$ \\
\hline$<2$ & 21 & $2.38 \pm 1.47$ & \\
\hline$\geq 2$ & 44 & $1.36 \pm 1.78$ & \\
\hline Clinical stage & & & 0.305 \\
\hline $\mathrm{I} / \mathrm{II}$ & 81 & $1.85 \pm 1.78$ & \\
\hline III/IV & 6 & $2.67 \pm 2.94$ & \\
\hline pT stage & & & 0.718 \\
\hline $\mathrm{T} 1 / \mathrm{T} 2$ & 32 & $1.81 \pm 1.58$ & \\
\hline $\mathrm{T} 3$ & 55 & $1.96 \pm 2.03$ & \\
\hline $\mathrm{pN}$ stage & & & 0.097 \\
\hline No & 75 & $2.28 \pm 1.86$ & \\
\hline N1 & 12 & $3.25 \pm 1.82$ & \\
\hline \multicolumn{4}{|l|}{ pM stage } \\
\hline M0 & 85 & $2.39 \pm 1.89$ & 0.411 \\
\hline M1 & 2 & $3.50 \pm 0.71$ & \\
\hline
\end{tabular}

${ }^{\mathrm{a}} \mathrm{P}<0.01$ and ${ }^{\mathrm{b}} \mathrm{P}<0.05$. Values are presented as the mean \pm standard deviation. SOX7, sex determining region Y-box 7; pT, primary tumor stage; $\mathrm{pN}$, regional lymph node stage; $\mathrm{pM}$, pathologic metastases stage.

present study demonstrated that there are reduced levels of SOX7 expression in liver and pancreatic cancer tissues, which may serve a suppressive role in the progression of these cancer types. To the best of our knowledge, this is the first study in which the association between the level of SOX7 expression and the clinicopathological features of liver and pancreatic cancers, particularly its prognostic significance, has been extensively investigated.

A previous study suggested that SOX genes are widely expressed and serve a role in the regulation of the $\mathrm{Wnt} / \beta$-catenin signaling pathway during development (23). A motif in the SOX7 protein enables it to bind to $\beta$-catenin, permitting their interaction (24), and so SOX7 is a key regulator in embryonic development. In addition, the $\mathrm{Wnt} / \beta$-catenin signaling pathway is known to have important roles in a number of malignancies, including lung, breast, colorectal, ovarian and liver cancer. A number of studies have revealed that SOX7 has an important role in tumorigenesis (10). In 2002, Katoh (17) demonstrated that SOX7 mRNA was significantly upregulated in esophageal cancer cell lines and in a primary gastric cancer cell line, however, it was downregulated in primary colorectal tumors, prostate cancer, lung cancer and breast cancer, indicating that the role of SOX7 in tumorigenesis may depend on the tumor type. To the best of our knowledge, the role of SOX7 in liver and pancreatic cancer has not been previously investigated, therefore the present study analyzed SOX7 protein levels in a human liver TMA (including 78 HCC tissues and 100 ICC tissues) and a pancreatic cancer TMA (containing 91 pancreatic cancer tissues and 5 adjacent non-cancerous pancreatic tissues). The results demonstrate that SOX7 expression levels decrease in liver and pancreatic cancers.

SOX7 expression was previously reported to be negatively correlated with the depth of invasion, lymph node metastasis, distant metastasis and pTNM stage in gastric cancer patients (25). Li et al (15) demonstrated that reduced SOX7 expression was significantly associated with poor differentiation $(\mathrm{P}=0.002)$, lymph node metastasis $(\mathrm{P}=0.011)$ and advanced TNM stage $(\mathrm{P}=0.006)$. Similarly, the present study investigated the association between the level of SOX7 protein and the clinicopathological features of the donors who were included in the TMAs. The results indicate that the downregulation of SOX7 expression levels in liver cancer was markedly associated with advanced clinical stage, $\mathrm{pT}$ stage and pN stage. Notably, these findings were further validated using the TCGA 
Table IV. Prognostic value of SOX7 mRNA expression for biochemical recurrence-free survival evaluated by the Cox proportional hazards model.

A, BCR free survival univariate analysis

\begin{tabular}{lll}
\hline Variable & HR $(95 \%$ CI & P-value \\
\hline Age (<60 vs. $\geq 60$ years) & $0.750(0.349-1.614)$ & 0.462 \\
Gender (male vs. female) & $0.615(0.314-1.202)$ & 0.155 \\
Clinical stage (I vs. II-IV) & $1.754(0.804-3.789)$ & 0.159 \\
pT stage (T1 vs. T2-T4) & $1.889(0.919-3.884)$ & 0.084 \\
pN stage (N0 vs. N1) & $0.893(0.119-6.682)$ & 0.912 \\
pM stage (M0 vs. M1) & $5.454(1.204-24.709)$ & $0.028^{\mathrm{a}}$ \\
SOX7 (low vs. high expression) & $0.499(0.251-0.991)$ & $0.047^{\mathrm{a}}$ \\
\hline
\end{tabular}

$\mathrm{B}, \mathrm{BCR}$ free survival multivariate analysis

Age (<60 vs. $\geq 60$ years)

Gender (male vs. female)

pM stage (M0 vs. M1)

SOX7 (low vs. high expression)

$$
\begin{gathered}
0.597(0.221-1.610) \\
0.895(0.336-2.185) \\
10.922(1.897-62.883) \\
0.314(0.130-0.760)
\end{gathered}
$$

0.308

0.807

$0.007^{\mathrm{b}}$

$0.010^{\mathrm{a}}$

${ }^{\mathrm{a}} \mathrm{P}<0.05$ and ${ }^{\mathrm{b}} \mathrm{P}<0.01$. SOX7, sex determining region Y-box 7; BCR, biochemical recurrence; HR, hazard ratio; CI, confidence interval; $\mathrm{pT}$, primary tumor stage; $\mathrm{pN}$, regional lymph node stage; $\mathrm{pM}$, pathologic metastases stage.

A

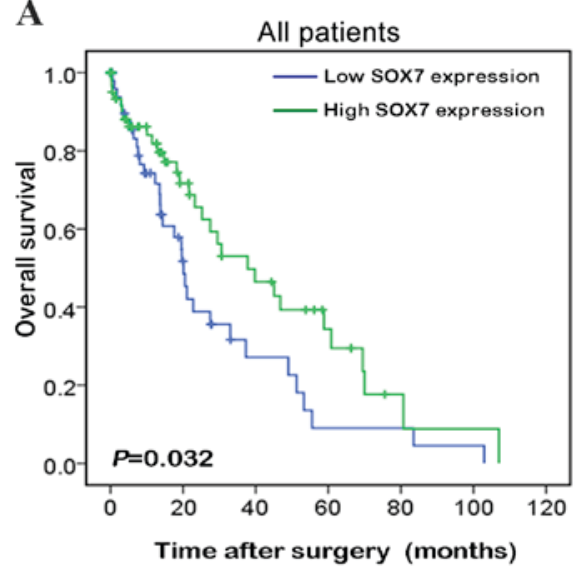

C

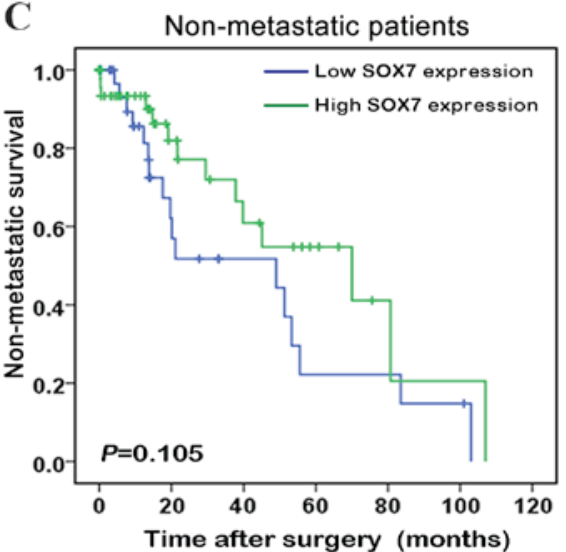

B

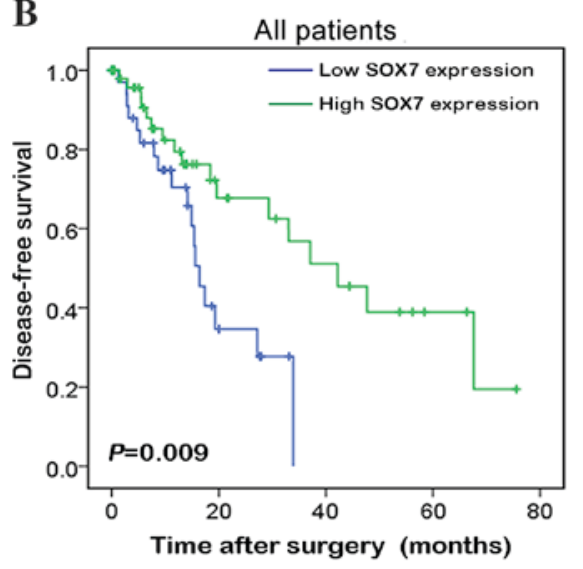

D

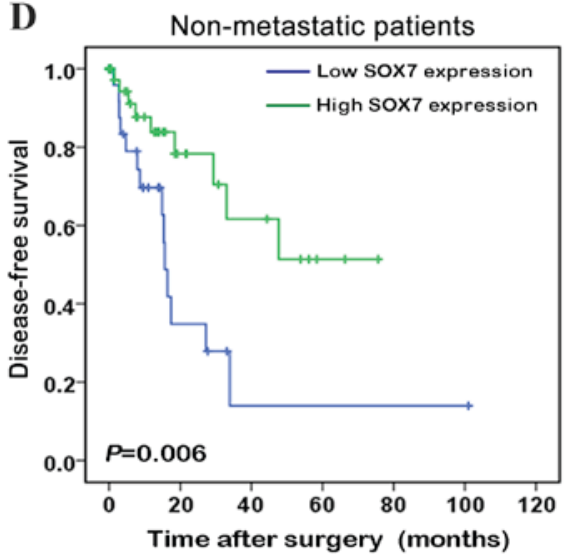

Figure 3. Decreased expression of SOX7 predicts poor prognosis in patients with liver cancer. The Kaplan-Meier method was used to evaluate the difference in (A) patient overall survival, (B) patient overall BCR (disease-free) survival, (C) non-metastatic patient survival and (D) non-metastatic patient BCR (disease-free) survival between the patients with high and low expression levels of SOX7. The mean of the SOX7 high-throughput sequencing mRNA expression levels in liver cancer tissues from the TCGA dataset was used as the cut-off to divide the liver cancer tissues from each patient into the high and low SOX7 expression groups. SOX7, sex determining region Y-box 7; BCR, biochemical recurrence. 
dataset. However, in the pancreatic cancer TMA dataset, SOX7 expression levels were not associated with advanced clinical stage or pTNM stage; it was only significant associated with the pathological grade of pancreatic cancer. These results support the hypothesis that the loss of SOX7 activity may critically contribute to cancer severity.

Previous studies have demonstrated that SOX7 expression in lung, prostate and gastric cancers is associated with the prognosis or survival of patients $(14,15,19,25)$. In the present study, due to the limited number of clinical specimens and follow-ups available for patients with liver cancer, the prognostic value of SOX7 was assessed based only on the TCGA dataset, which consisted of 134 liver cancer tissues with high-throughput sequencing data for mRNA expression. The results suggest that decreased expression of SOX7 is associated with poor prognosis in patients with liver cancer. The overall survival and the disease-free survival were shorter in patients with low SOX7 expression cancer types than in those with high SOX7 expression cancer types. These results are consistent with previous work (19), suggesting that the reduced expression of SOX7 may be a potential biomarker for poor prognosis in patients with liver cancer.

Previous evidence suggests that SOX7 may have tumor suppressive functions in a number of cancer types (14-16). Multiple reports have indicated that SOX7 inhibits proliferative $\beta$-catenin signaling via direct protein interactions, suggesting that it antagonizes tumor growth, at least in part, through activities that are independent of DNA binding (25). Takash et al (24) demonstrated that SOX7 inhibits TCF/LEF- $\beta$-catenin activation of the TCF/LEF-dependent TOPFLASH reporter in HEK293 cells, an observation that was later recapitulated in colon and prostate cancer cell lines $(14,26)$. In addition, SOX7 co-localizes and physically interacts with $\beta$-catenin in endometrial cancer cells (16).

In conclusion, the results suggest that the decreased expression of SOX7 is an important feature of liver cancer. The expression levels of the SOX7 protein may be a potential indicator for predicting the clinical outcome of patients with liver cancer. The results of the present study may significantly contribute to the understanding of the molecular mechanisms underlying carcinogenesis and disease progression in liver cancer.

\section{Acknowledgements}

The present study was supported by the Science and Technology Project of Huadu District of Guangzhou (grant no. 15-HDWS-012).

\section{References}

1. McGlynn KA, Tsao L, Hsing AW, Devesa SS and Fraumeni JF Jr: International trends and patterns of primary liver cancer. Int $\mathbf{J}$ Cancer 94: 290-296, 2001.

2. Mittal S and El-Serag HB: Epidemiology of hepatocellular carcinoma: Consider the population. J Clin Gastroenterol 47 (Suppl): S2-S6, 2013

3. Wolfgang CL, Herman JM, Laheru DA, Klein AP, Erdek MA, Fishman EK and Hruban RH: Recent progress in pancreatic cancer. CA Cancer J Clin 63: 318-348, 2013.

4. Bosman FT, Carneiro F, Hruban RH and Theise ND: WHO classification of tumours of the digestive system. World Health Organization, Geneva, 2010.
5. Jemal A, Bray F, Center MM, Ferlay J, Ward E and Forman D: Global cancer statistics. CA Cancer J Clin 61: 69-90, 2011.

6. Wegner M: From head to toes: The multiple facets of Sox proteins. Nucleic Acids Res 27: 1409-1420, 1999.

7. Stovall DB, Cao P and Sui G: SOX7: From a developmental regulator to an emerging tumor suppressor. Histol Histopathol 29: 439-445, 2014.

8. Gandillet A, Serrano AG, Pearson S, Lie-A-Ling M, Lacaud G and Kouskoff V: Sox7-sustained expression alters the balance between proliferation and differentiation of hematopoietic progenitors at the onset of blood specification. Blood 114: 4813-4822, 2009.

9. Nelson TJ, Chiriac A, Faustino RS, Crespo-Diaz RJ, Behfar A and Terzic A: Lineage specification of Flk-1+ progenitors is associated with divergent Sox7 expression in cardiopoiesis. Differentiation 77: 248-255, 2009.

10. Cermenati S, Moleri S, Cimbro S, Corti P, Del Giacco L, Amodeo R, Dejana E, Koopman P, Cotelli F and Beltrame M: Sox18 and Sox7 play redundant roles in vascular development. Blood 111: 2657-2666, 2008.

11. Séguin CA, Draper JS, Nagy A and Rossant J: Establishment of endoderm progenitors by SOX transcription factor expression in human embryonic stem cells. Cell Stem Cell 3: 182-195, 2008.

12. Savage J, Conley AJ, Blais A and Skerjanc IS: SOX15 and SOX7 differentially regulate the myogenic program in P19 cells. Stem Cells 27: 1231-1243, 2009.

13. Wang C, Guo Y, Wang J and Min Z: The suppressive role of SOX7 in hepatocarcinogenesis. PLoS One 9: e97433, 2014.

14. Zhang Y, Huang S, Dong W, Li L, Feng Y, Pan L, Han Z, Wang X, Ren G, Su D, et al: SOX7, down-regulated in colorectal cancer, induces apoptosis and inhibits proliferation of colorectal cancer cells. Cancer Lett 277: 29-37, 2009.

15. Li B, Ge Z, Song S, Zhang S, Yan H, Huang B and Zhang Y: Decreased expression of SOX7 is correlated with poor prognosis in lung adenocarcinoma patients. Pathol Oncol Res 18: 1039-1045, 2012.

16. Chan DW, Mak CS, Leung TH, Chan KK and Ngan HY: Down-regulation of sox7 is associated with aberrant activation of wnt/b-catenin signaling in endometrial cancer. Oncotarget 3 : 1546-1556, 2012.

17. Katoh M: Expression of human SOX7 in normal tissues and tumors. Int J Mol Med 9: 363-368, 2002.

18. Stovall DB, Wan M, Miller LD, Cao P, Maglic D, Zhang Q, Stampfer MR, Liu W, Xu J and Sui G: The regulation of SOX7 and its tumor suppressive role in breast cancer. Am J Pathol 183: 1645-1653, 2013.

19. Zhong WD, Qin GQ, Dai QS, Han ZD, Chen SM, Ling XH, Fu X, Cai C, Chen JH, Chen XB, et al: SOXs in human prostate cancer: Implication as progression and prognosis factors. BMC Cancer 12: 248, 2012.

20. Qi J, Wang J, Katayama H, Sen S and Liu SM: Circulating microRNAs (cmiRNAs) as novel potential biomarkers for hepatocellular carcinoma. Neoplasma 60: 135-142, 2013.

21. Zhang Y, Jiang H, Qin M, Su X, Cao Z and Wang J: TNIK serves as a novel biomarker associated with poor prognosis in patients with pancreatic cancer. Tumour Biol 37: 1035-1040, 2016.

22. Wong $R$ and Frenette $C$ : Updates in the management of hepatocellular carcinoma. Gastroenterol Hepatol (N Y) 7: 16-24, 2011.

23. Kormish JD, Sinner D and Zorn AM: Interactions between SOX factors and Wnt/beta-catenin signaling in development and disease. Dev Dyn 239: 56-68, 2010.

24. Takash W, Cañizares J, Bonneaud N, Poulat F, Mattéi MG, Jay P and Berta P: SOX7 transcription factor: Sequence, chromosomal localisation, expression, transactivation and interference with Wnt signalling. Nucleic Acids Res 29: 4274-4283, 2001.

25. Cui J, Xi H, Cai A, Bian S, Wei B and Chen L: Decreased expression of Sox7 correlates with the upregulation of the Wnt $/ \beta$-catenin signaling pathway and the poor survival of gastric cancer patients. Int J Mol Med 34: 197-204, 2014.

26. Guo L, Zhong D, Lau S, Liu X, Dong XY, Sun X, Yang VW, Vertino PM, Moreno CS, Varma V, et al: Sox7 is an independent checkpoint for beta-catenin function in prostate and colon epithelial cells. Mol Cancer Res 6: 1421-1430, 2008. 